\title{
Sensory Deprivation Alters Aggrecan and Perineuronal Net Expression in the Mouse Barrel Cortex
}

\author{
Paulette A. McRae, ${ }^{1}$ Mary M. Rocco, ${ }^{2}$ Gail Kelly, ${ }^{1}$ Joshua C. Brumberg, ${ }^{2,3}$ and Russell T. Matthews ${ }^{1,4}$ \\ ${ }^{1}$ Department of Neurobiology, Yale University School of Medicine, New Haven, Connecticut 06520, ${ }^{2}$ Neuropsychology PhD Subprogram, The Graduate \\ Center, City University of New York (CUNY), New York, New York 10016, ${ }^{3}$ Department of Psychology, Queens College, CUNY, Flushing, New York 11367, \\ and ${ }^{4}$ Department of Neuroscience and Physiology, State University of New York Upstate Medical University, Syracuse, New York 13210
}

An important role for the neural extracellular matrix in modulating cortical activity-dependent synaptic plasticity has been established by a number of recent studies. However, identification of the critical molecular components of the neural matrix that mediate these processes is far from complete. Of particular interest is the perineuronal net (PN), an extracellular matrix component found surrounding the cell body and proximal neurites of a subset of neurons. Because of the apposition of the PN to synapses and expression of this structure coincident with the close of the critical period, it has been hypothesized that nets could play uniquely important roles in synapse stabilization and maturation. Interestingly, previous work has also shown that expression of PNs is dependent on appropriate sensory stimulation in the visual system. Here, we investigated whether PNs in the mouse barrel cortex are expressed in an activity-dependent manner by manipulating sensory input through whisker trimming. Importantly, this manipulation did not lead to a global loss of PNs but instead led to a specific decrease in PNs, detected with the antibody Cat-315, in layer IV of the barrel cortex. In addition, we identified a key activity-regulated component of PNs is the proteoglycan aggrecan. We also demonstrate that these Cat-315-positive neurons virtually all also express parvalbumin. Together, these data are in support of an important role for aggrecan in the activity-dependent formation of PNs on parvalbumin-expressing cells and suggest a role for expression of these nets in regulating the close of the critical period.

Key words: chondroitin sulfate; lectican; extracellular matrix; proteoglycan; parvalbumin; somatosensory cortex; critical period

\section{Introduction}

The role of experience or activity in shaping neural development and plasticity has been intensely investigated since the landmark work of Wiesel and Hubel (1963a,b) in the visual cortex. As their work and subsequent studies have clearly demonstrated, the circuitry in the CNS is shaped by experience early in development during a so-called "critical period." The formation and stabilization of synaptic connections in the CNS involves the complex interplay between the developing neuronal cell surface and the molecules in the extracellular space. Because of its role in organizing the extracellular space, the neural extracellular matrix (ECM) is thought to play particularly important roles in these processes. The perineuronal net $(\mathrm{PN})$, a subcomponent of the neural ECM, is found surrounding synapses on the cell body and proximal neurites of certain neurons in a lattice-like structure and is therefore uniquely positioned to influence synaptic development and stabilization (Hockfield et al., 1990; Celio and Blumcke, 1994).

\footnotetext{
Received Dec. 15, 2006; revised March 7, 2007; accepted April 11, 2007.

This work was supported by National Institute of Neurological Disorders and Stroke Grant NS 007224-21S1 and the American Psychological Association Diversity Fellowship. We thank Svetlana Abrams, Eric Chen, Sid Dave, Danny Tam, and Ferdous Zanat for assistance with whisker trimming and tissue preparation. We also thank Shyron Alston for technical help on this project.

Correspondence should be addressed to Dr. Russell T. Matthews, Department of Neuroscience and Physiology, WH 3238, State University of New York Upstate Medical University, Syracuse, NY 13210. E-mail: matthewr@upstate.edu.

DOI:10.1523/JNEUROSCI.5425-06.2007

Copyright $\odot 2007$ Society for Neuroscience $\quad$ 0270-6474/07/275405-09\$15.00/0
}

In the cat visual system, PNs detected with the monoclonal antibody Cat-301 appear in postnatal development, coincident with a decrease in plasticity and the close of the critical period (Sur et al., 1988). The temporal appearance of PNs implies that they play a role in maintaining synaptic stability in the mature animal (Zaremba et al., 1989). Sensory deprivation early in development leads to decreased PN expression in the visual system (Sur et al., 1988; Guimaraes et al., 1990; Kind et al., 1995; Lander et al., 1997) but has no effect in the adult (Sur et al., 1988). These results suggest that $\mathrm{PN}$ expression is dependent on appropriate sensory stimulation.

Whereas work in the cat visual cortex suggests an important role for PNs in developmental cortical plasticity, a thorough understanding of the molecular composition of these nets and how these molecules are altered by activity is incomplete. Particularly important components of the PN are members of a family of chondroitin sulfate proteoglycans (CSPGs) named the lecticans (Yamaguchi, 2000). Although all lectican family members can be found in PNs, it is unknown whether expression of any particular lectican is altered by activity.

To more thoroughly investigate the roles of PNs and specific $\mathrm{PN}$ components in cortical developmental plasticity, we used a mouse model system that allowed us to more comprehensively investigate these questions. Because developmental plasticity is well established in the rodent barrel cortex (Fox, 2002), we used this system to gain additional insight into the role of PNs in developmental cortical plasticity. Our preliminary studies dem- 
onstrated that PNs are highly enriched in the mouse barrel cortex. Our work suggests a uniquely important role for the activitydependent expression of PNs on a subset of interneurons in layer IV of the mouse barrel cortex. Furthermore, we identify the lectican aggrecan as a key activity-regulated component of the $\mathrm{PN}$ in the mouse whisker barrel cortex. These data suggest that aggrecan expression and PN expression may have an important and specific role in activity-dependent plasticity in the rodent somatosensory cortex.

\section{Materials and Methods}

Animals and experimental groups. Experimental animals, CD-1 mice (Charles River Laboratories, Wilmington, MA), were obtained from pregnant dams. Mice were housed together in standard plastic cages with woodchip bedding and ad libitum access to food and water. Littermates were randomly assigned into the control group or the experimental group, whose whiskers were trimmed. There were three sensorydeprivation paradigms used. The day of birth was designated postnatal day $0(\mathrm{P} 0)$. To look at the effects of developmental sensory deprivation, the whiskers were trimmed from P0 to P30 [11 controls and 10 trimmed animals for Cat-315 analysis and 8 animals for Wisteria floribunda agglutinin (WFA) analysis]. To study the effect of sensory deprivation in adulthood, animals had their whiskers trimmed from P90 to P120 (four control animals and five trimmed animals). To understand whether any potential deficits that occurred from $30 \mathrm{~d}$ of trimming could be reversed, a group had their whiskers trimmed from P0 to P30, and the whiskers were allowed to regrow and have normal sensory input from P30 to P60 (nine regrow animals).

Sensory deprivation. The large mystacial whiskers (rows A-E and arcs $0-6$ ) were trimmed within $1 \mathrm{~mm}$ of the skin on the right side of the face every other day using microsurgical spring scissors (World Precision Instruments, Sarasota, FL). During the first $12 \mathrm{~d}$, the mice were restrained by holding them in the experimenter's hand. After P13, the animals were anesthetized, using isoflurane (Aerrane) for 1-3 min delivered through a precision vaporizer (Precision Medical, Northampton, PA) at $0.25 \%$ to prevent movement and spontaneous whisking, which interferes with the trimming process. Control animals were handled and anesthetized at the same time as the sensory-deprived animals but did not have their whiskers trimmed.

Immunohistochemistry. Mice were deeply anesthetized and perfused transcardially with $0.1 \mathrm{M}$ PBS, followed by $4 \%$ phosphate-buffered paraformaldehyde, $\mathrm{pH}$ 7.4. The tissue was postfixed overnight with $30 \%$ sucrose in phosphate buffer. Forty to $50 \mu \mathrm{m}$ frozen sections were cut on a cryostat. Free-floating sections were incubated at $4^{\circ} \mathrm{C}$ overnight in primary antibodies Cat-315, parvalbumin (Sigma, St. Louis, MO), or somatostatin (Chemicon, Temecula, CA) with $0.5 \%$ Triton X-100. The following day, they were rinsed with phosphate buffer and incubated at room temperature in HRP-conjugated goat anti-mouse secondary antibodies (Jackson ImmunoResearch, West Grove, PA) or Alexa fluorescent-conjugated goat anti-mouse secondary antibodies (Invitrogen, Eugene, OR) diluted in DMEM with $0.5 \%$ fetal calf serum and $0.5 \%$ Triton X-100 for $2 \mathrm{~h}$. Fluorescent Nissl (Invitrogen) and WFA (Vector Laboratories, Burlingame, CA) were added at the same time as the secondary antibodies. Sections were rinsed in phosphate buffer, mounted onto glass slides, and coverslipped using Prolong Antifade mounting medium (Invitrogen).

In situ hybridization. Frozen brains sectioned coronally or tangentially (15-20 $\mu \mathrm{m}$ thick) were thaw mounted onto gelatin-coated slides and postfixed in $0.1 \mathrm{M}$ sodium phosphate-buffered $4 \%$ paraformaldehyde, $\mathrm{pH}$ 7.4. Sections were rinsed in PBS and $2 \times$ SSC and acetylated with $0.5 \%$ acetic anhydride in $0.1 \mathrm{~m}$ triethanolamine, $\mathrm{pH} 8.0$. Sections were rinsed in $2 \times$ SSC and PBS, dehydrated in ethanols, and delipidated in chloroform. Sections were prehybridized in $2 \times$ SSC and $50 \%$ formamide at $55^{\circ} \mathrm{C}$ for $1 \mathrm{~h}$. Sections were then hybridized in $0.75 \mathrm{M} \mathrm{NaCl}, 50 \%$ formamide, $1 \times$ Denhardt's solution, $10 \%$ dextran sulfate, $30 \mathrm{~mm}$ dithiothreitol, $10 \mathrm{~mm}$ Tris-HCl, pH 7.5, 1 mм EDTA, $100 \mu \mathrm{g} / \mathrm{ml}$ salmon sperm DNA, 0.5 $\mathrm{mg} / \mathrm{ml}$ yeast tRNA, and $1.5 \times 10^{6} \mathrm{cpm}$ probe per slide for $12-15 \mathrm{~h}$ at $55^{\circ} \mathrm{C}$. $\left[{ }^{33} \mathrm{P}\right]$-labeled aggrecan probe to exon 12 was synthesized using an
SP6/T7 transcription kit (Hoffmann-La Roche, Nutley, NJ). After hybridization, the sections were washed in $2 \times$ SSC, $50 \%$ formamide, and $0.1 \% \beta$-mercaptoethanol (BME) at $55^{\circ} \mathrm{C}$ for $1 \mathrm{~h}$ and treated with 20 $\mu \mathrm{g} / \mathrm{ml} \mathrm{RNase} \mathrm{A}$ in $0.5 \mathrm{M} \mathrm{NaCl}$ and $10 \mathrm{~mm}$ Tris- $\mathrm{HCl}, \mathrm{pH} 8.0$, at $37^{\circ} \mathrm{C}$ for 30 $\mathrm{min}$. The sections were then washed in $2 \times$ SSC, $50 \%$ formamide, and $0.1 \% \mathrm{BME}$ at $60^{\circ} \mathrm{C}$ for $30 \mathrm{~min}$ and in $0.1 \times \mathrm{SSC}$ and $0.1 \% \mathrm{BME}$ at $65^{\circ} \mathrm{C}$ for $30 \mathrm{~min}$ and dehydrated. The slides were exposed to film (Kodak BioMax MR Film; Eastman Kodak, Rochester, NY) for $11 \mathrm{~d}$. Autoradiograms were scanned into Photoshop and used as inverted images. Sections used for autoradiograms were counterstained with cresyl violet to demarcate barrel regions.

Scanned autoradiograms were analyzed using ImageJ software to determine the relative level aggrecan mRNA by densitometry. Layers IV and VI of the barrel cortex were manually defined, and the density of signals was then calculated. The data were analyzed comparing the intensity of signal per unit area in layer IV of the deprived barrel cortex to layer IV of the nondeprived barrel cortex and layer VI deprived barrel cortex to layer VI of the nondeprived barrel cortex. A paired $t$ test was used to evaluate levels of expression in layers IV and VI of the barrel cortex. The experimenter was blinded to the experimental conditions.

Stereology. Numerical densities were estimated using the optical fractionator method (West et al., 1991). Counts were performed on an Olympus (Tokyo, Japan) BX51W microscope with a motorized stage. Stereo Investigator software (MicroBrightField, Williston, VT) was used to analyze serial sections ( $40-50 \mu \mathrm{m}$ thick) cut tangentially through layer IV of the barrel cortex or coronally to analyze layer VI. The contours of the barrels in layer IV of the cortex were outlined in the Stereo Investigator program using the $4 \times$ lens, and the number of cells marked with the primary antibodies were counted using an oil-immersion $20 \times$ lens (0.8 numerical aperture) and appropriate excitation and emission filters. A minimum of nine random sampling sites were analyzed throughout the depth of the tissue, excluding $4 \mu \mathrm{m}$ for the guard zones ( $2 \mu \mathrm{m}$ at the top of the section and $2 \mu \mathrm{m}$ at the bottom). Stereo Investigator software was used to quantify the number of marked cells and provided an estimate of total cell number in the tissue section. The number of cells, denoted by the fluorescent Nissl, was also counted using the same methods. In all cases, the experimenter was blinded to the experimental conditions.

Statistical analysis. All sensory-deprivation paradigms were analyzed using a paired $t$ test for each group of animals, comparing layer IV and VI of the deprived barrel cortex to the respective layers of the nondeprived barrel cortex. For the P30 sensory-deprived animals and the adult sensory-deprived animals, a two-way repeated-measures ANOVA was also performed.

\section{Results}

\section{Cat-315-positive PNs are expressed in the mouse barrel cortex}

Previous work has shown that developmental expression of PNs depends on normal sensory input and activity in the cat visual cortex (Guimaraes et al., 1990; Lander et al., 1997). Although PNs in the visual system have been well studied, the expression of this structure is not well understood in other sensory systems. Here, we investigated the expression of PNs in the mouse barrel cortex. Our preliminary studies using the monoclonal antibody Cat-315, an antibody that recognizes a specific form of aggrecan in the adult brain (Matthews et al., 2002), demonstrated that PNs are highly expressed in the barrel cortex. Cat-315 was enriched in layers IV and VI of the mouse cortex (Fig. 1A). Cat-315 staining was more abundant in the somatosensory cortex than surrounding cortical areas (Fig. $1 \mathrm{~B}$ ), where it detects the $\mathrm{PN}$ around the cell body and proximal neurites of a subset of neurons. In additional studies, we evaluated Cat-315 expression specifically in layer IV of the mouse barrel cortex because it is the first layer of the cortex to receive and respond to input from the whiskers and PN properties could be observed in the context of the functional bound- 

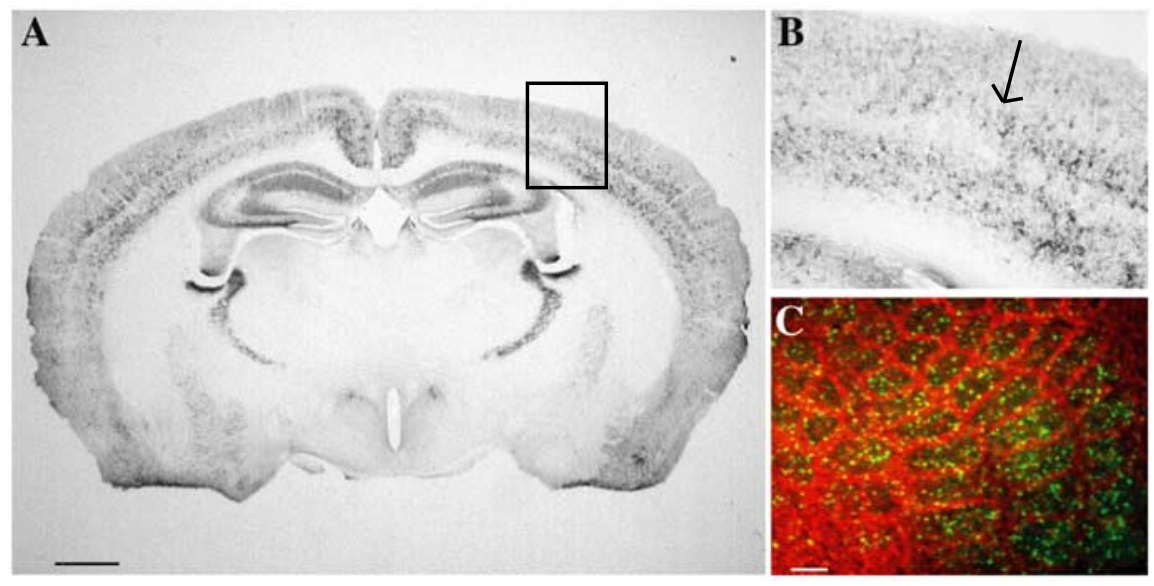

Figure 1. Monoclonal antibody Cat-315 expression in the mouse somatosensory cortex. Cat-315 marks a subset of cells within the barrel region of the mouse. $A$, A coronal section shows that Cat- 315 is more enriched in layers IV and VI of the somatosensory cortex than in adjacent cortical areas. $\boldsymbol{B}$, Higher magnification of the boxed region in $\boldsymbol{A}$ demonstrates high expression of Cat-315reactive nets in the somatosensory cortex (arrow denotes the border). $C$, A tangential section through layer IV of the barrel cortex showing (at-315 (green) immunoreactivity is highly expressed within the boundaries of the barrel cortex, defined by fluorescent Nissl (red). Scale bars: $A, 1 \mathrm{~mm} ; \boldsymbol{C}, 200 \mu \mathrm{m}$.

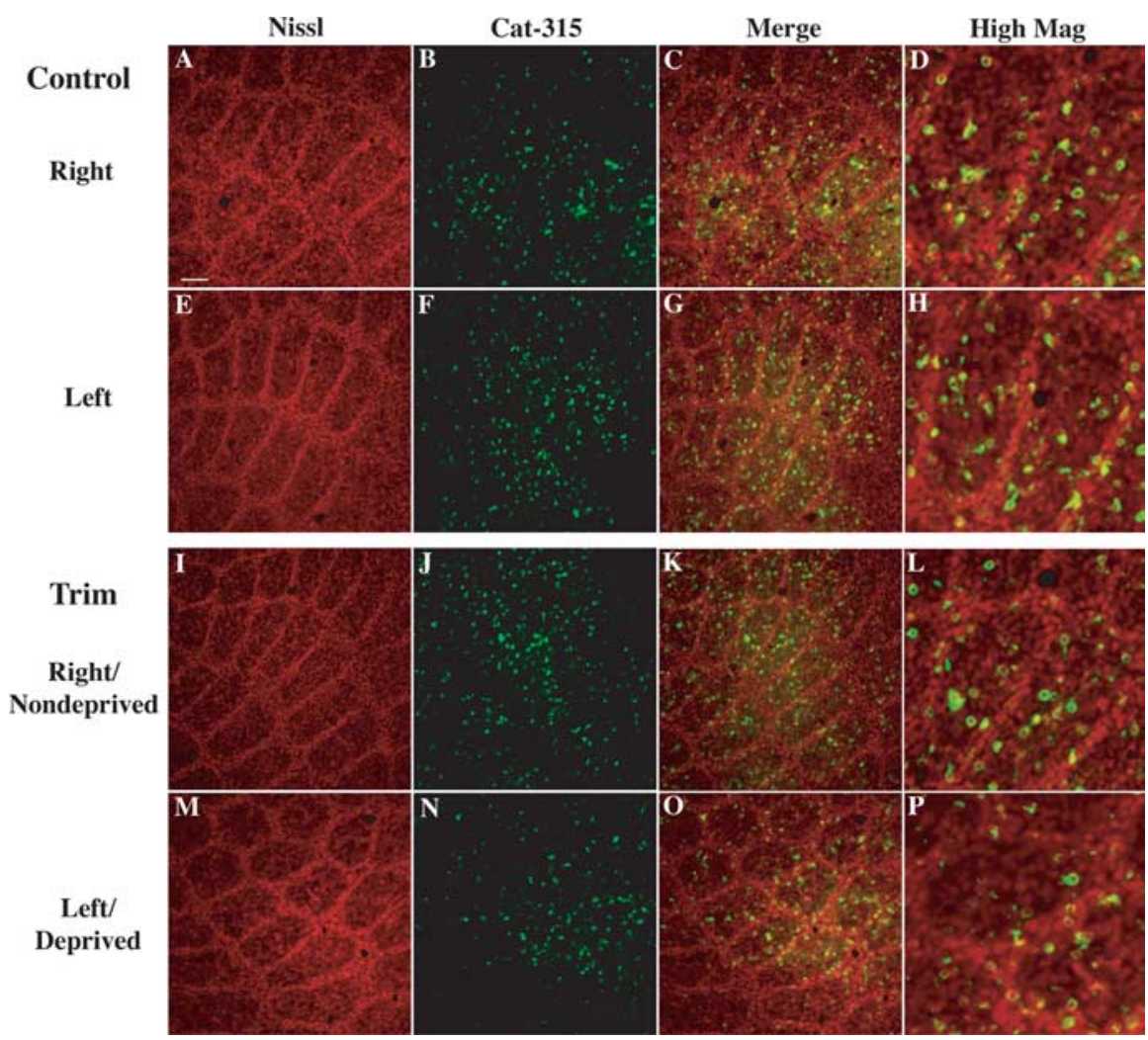

Figure 2. Effects of sensory deprivation on Cat-315-positive PNs in the mouse barrel cortex. Tangential sections through layer IV of the barrel cortex of control $(\boldsymbol{A}-\boldsymbol{H})$ and sensory-deprived $(\boldsymbol{I}-\boldsymbol{P})$ mice stained with fluorescent Niss $(\boldsymbol{A}, \boldsymbol{E}, \boldsymbol{I}, \boldsymbol{M})$ and Cat-315 $(\boldsymbol{B}$, $\boldsymbol{F}, \boldsymbol{J}, \boldsymbol{N})$ are shown. $\boldsymbol{C}, \boldsymbol{G}, \boldsymbol{K}$, and $\mathbf{O}$ show the merge of the fluorescent Nissl (red) and Cat-315 (green). $\boldsymbol{D}, \boldsymbol{H}, \boldsymbol{L}$, and $\boldsymbol{P}$ are higher-magnification images of $\boldsymbol{C}, \boldsymbol{G}, \boldsymbol{K}$, and $\boldsymbol{O}$. The right hemisphere $(\boldsymbol{A}-\boldsymbol{D})$ did not differ from the left hemisphere $(\boldsymbol{E}-\boldsymbol{H})(\boldsymbol{p}=$ 0.3878). Unilateral whisker trimming from the right whisker pad did not alter barrel morphology $(I, M)$ but did substantially reduce Cat-315 immunoreactivity in the sensory-deprived hemisphere $(\boldsymbol{N}-\boldsymbol{P})$ compared with the nondeprvied hemisphere $(\boldsymbol{J}-\boldsymbol{L})$. The level of reduction can also be observed by comparing Cat-315 levels in the deprived barrel cortex with those of control animals $(\boldsymbol{B}-\boldsymbol{D}, \boldsymbol{F}-\boldsymbol{H})$. Quantification of these data are shown in Table 1. Scale bar, $100 \mu \mathrm{m}$.

aries of the barrels. In tangential sections through layer IV, Cat315 was localized inside the barrel cortex boundaries (Fig. 1C). Together, these data indicate that PNs are highly expressed in the barrel cortex and that aggrecan, detected with Cat-315, is a major component of PNs in the mouse barrel cortex. The expression of Cat-315-positive PNs suggest an important role for nets in this region.

Sensory deprivation during the first $30 \mathrm{~d}$ of development leads to decreased Cat315 expression in layer IV of the barrel cortex

Because aggrecan-reactive nets are strongly expressed in the postnatal barrel cortex (Fig. $2 A-H)$, we asked whether altering sensory input from the whiskers would alter the expression of PNs and aggrecan. To evaluate this, whiskers were trimmed from the right whisker pad of mice every other day from birth through P30. Tangential sections through layer IV of the barrel cortex were analyzed using stereological methods. Nissl staining of the barrel cortex illustrated that the gross development of the barrels was not altered by this manipulation and did not differ from controls (Fig. 2A,E,I,M). However, our studies revealed a significant decrease in the number of cells with Cat315-positive PNs in the sensory-deprived barrel cortex of trimmed animals (Fig. $2 N-P$ ) compared with the nondeprived barrel cortex of the same animals (Fig. $2 J-L)$ and compared with both barrel cortices of control animals (Fig. $2 B-D, F-H$; Table 1). There was a statistically significant reduction in Cat-315 staining in trimmed animals compared with control animals using a two-way repeatedmeasures ANOVA ( $p=0.0282)$. The decrease in Cat-315 in the deprived hemisphere compared with the nondeprived hemisphere in trimmed animals was significant using a paired $t$ test $(p=0.0034)$ (Table 1). There was also a significant decrease in the deprived hemisphere of trimmed animals compared with the left hemisphere of control animals ( $p=$ 0.0015) (Table 1). However, the nondeprived barrel cortex did not differ significantly from the barrel cortex of control animals ( $p=0.9454$ ) (Table 1), therefore the nondeprived barrel cortex of trimmed animals will be used as an internal control. As a result of sensory deprivation, there was a $22 \%$ decrease in the number of neurons having Cat-315-positive PNs in the deprived barrel cortex compared with the nondeprived barrel cortex of trimmed animals. There was no difference in the total number of cells in the barrel cortex of trimmed animals compared with controls ( $p=0.3477)$. In addition, we found no significant changes in Cat-315 expression in layer VI of the barrel cortex (data not shown). These data demonstrate that within layer IV, aggrecan expression and $\mathrm{PN}$ formation is activity dependent and requires normal sensory stimulation during development. 
Table 1. Stereological cell counts of percentage of Cat-315 positive to total cell number

\begin{tabular}{lllll}
\hline Group & $n$ & $\begin{array}{l}\text { Nondeprived (right), percentage of cells that are } \\
\text { Cat-315 }+( \pm S D)\end{array}$ & $\begin{array}{l}\text { Deprived (left), percentage of cells that are } \\
\text { Cat-315 }+( \pm S D)\end{array}$ & $p$ value (right vs left) \\
\hline P30 control & 11 & $7.419125 \pm 1.851511$ & $7.589479 \pm 1.462587$ & 0.3878 \\
P30 Trim & 10 & $7.395954 \pm 1.78214$ & $5.769441 \pm 1.304703^{*}$ & 0.0034 \\
Adult control & 4 & $5.598945 \pm 0.682995^{* *}$ & $5.67208 \pm 0.940417^{* *}$ & 0.8708 \\
Adult Trim & 5 & $6.845911 \pm 0.697807$ & $6.768525 \pm 0.794268$ & 0.8673 \\
Regrow & 8 & $6.352756 \pm 1.841329$ & $3.286938 \pm 1.622181^{* * *}$ & 0.0003 \\
\hline
\end{tabular}

Trim, Trimmed animals. ${ }^{*} p=0.0015$ compared with left hemisphere of P30 control; ${ }^{* *} p<0.05$ compared with same hemisphere of P30 control; ${ }^{* * *} p=0.0014$ compared with deprived hemisphere of P30 Trim.

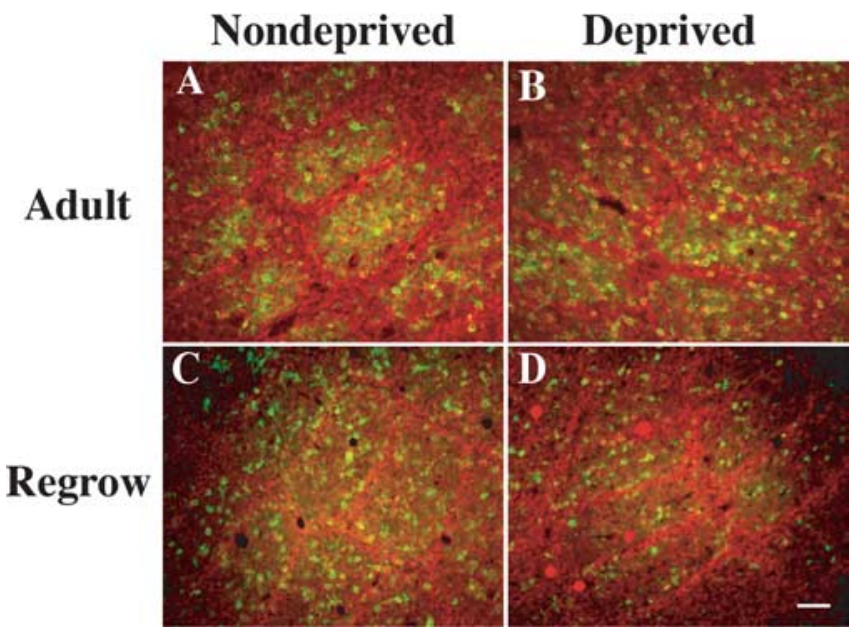

Figure 3. Effects of sensory deprivation in adulthood, or sensory deprivation followed by normal activity, on Cat-315-expressing PNs in the mouse barrel cortex. $\boldsymbol{A}, \boldsymbol{B}$, Tangential sections through layer IV of the nondeprived barrel cortex $(\boldsymbol{A})$ and sensory-deprived barrel cortex $(\boldsymbol{B})$ of adult mice after sensory deprivation from P90 to P120 did not alter Cat-315 expression (green) or fluorescent Nissl staining (red). C, D, Sensory deprivation through P30 followed by $30 \mathrm{~d}$ of normal activity did not alter barrel morphology, as shown by the fluorescent Nissl staining, but did significantly reduce Cat-315 immunoreactivity in the sensory-deprived hemisphere (D) compared with the nondeprvied hemisphere (C) (see Table 1). Scale bar, $100 \mu \mathrm{m}$.

\section{Sensory deprivation during adulthood does not alter Cat-315} expression in layer IV of the barrel cortex

Next, we asked whether the number of cells with Cat-315positive PNs could be altered by sensory activity in adulthood. The whiskers of adult mice were unilaterally trimmed from the right whisker pad for $30 \mathrm{~d}$ starting at P90. Whisker trimming in these animals did not alter Cat-315-positive PN expression in the deprived barrel cortex compared with the nondeprived barrel cortex within the same animals (Fig. $3 A, B$; Table 1), and there was no difference in the number of cells expressing Cat-315reactive $\mathrm{PNs}$ in trimmed animals compared with control animals $(p=0.9955)$. Therefore, normal sensory input from the whiskers is not required for maintenance of Cat-315-positive PNs once they have been established. This implies that there is a critical period for PN development that requires normal sensory input before P90.

\section{PN expression in the barrel cortex depends on appropriate} activity within a critical period

To further define the critical period for Cat-315-reactive PN expression, we investigated the effect of whisker trimming for the first $30 \mathrm{~d}$ of postnatal life, after which the whiskers were allowed to regrow and receive normal stimulation for the next $30 \mathrm{~d}$. Animals were then killed and analyzed at day 60. Again, whisker trimming did not alter Nissl staining; however, there was a $48 \%$ decrease in cells expressing Cat-315-positive PNs in the deprived barrel cortex relative to the nondeprived barrel cortex (Fig. 3C,D;
Table 1). Sensory deprivation followed by a period of normal whisker stimulation led to a significant decrease in cells expressing Cat-315-reactive PNs in the deprived barrel cortex even after allowing the whiskers to regrow. It is of note that trimming the whiskers for $30 \mathrm{~d}$ alone leads to a $22 \%$ decrease in Cat-315expressing PNs (Fig. 2, Table 1), whereas allowing the whisker regrowth and stimulation $30 \mathrm{~d}$ after this manipulation leads to a more significant decrease in PN expression ( $p=0.0014$ ) (Table $1)$. In contrast, there was no significant decrease in the number of Cat-315-positive PNs on the nondeprived sides between P30 and P60, suggesting a specific continual loss of Cat-315-positive PNs in the deprived cortex (Table 1). Providing normal sensory input after $30 \mathrm{~d}$ of sensory deprivation did not lead to a recovery of normal Cat-315 detection of PNs. These data indicate that normal PN expression in layer IV of the barrel cortex requires sensory stimulation specifically during the first $30 \mathrm{~d}$ of life.

aggrecan mRNA is highly enriched in the barrel cortex

We have demonstrated that aggrecan protein expression, detected with the antibody Cat-315, is significantly decreased in the barrel cortex if there is decreased sensory activity for the first $30 \mathrm{~d}$ of life. Our previous work has shown that this reagent detects a specific glycoform of aggrecan protein on a subset of PNs in the cortex (Matthews et al., 2002). Therefore, it is critical to determine whether sensory deprivation leads to a specific loss in the glycoform of aggrecan detected by Cat-315 or a general loss of aggrecan protein. Presently, there is no reliable "pan-antibody" that detects all forms of aggrecan in PNs by immunohistochemistry, so to address this question, we analyzed the expression of aggrecan mRNA. First, we analyzed normal aggrecan mRNA expression within the barrel cortex. Antisense radiolabeled mouse aggrecan probes showed very strong labeling in tangential flattened sections of the barrel cortex. After the sections were developed, they were stained with cresyl violet to delineate the boundaries of the barrel cortex (Fig. 4A). Interestingly, expression of aggrecan mRNA delineated the barrel cortex in layer IV from the surrounding cortical areas (Fig. $4 B$ ). In coronal sections, the labeling in the barrel cortex was clearly higher than any adjacent cortical regions in layers IV and VI of mouse barrel cortex (Fig. $4 C)$. In layers IV and VI of the barrel cortex, aggrecan mRNA labeling pattern was similar to the aggrecan protein expression characterized by Cat-315 (Fig. 1). These data indicate that aggrecan mRNA is highly expressed in the barrel cortex and suggest a uniquely important role for this ECM molecule in the barrel cortex specifically within thalamocortical recipient zones.

\section{aggrecan mRNA is decreased in layer IV of the barrel cortex} after $30 \mathrm{~d}$ of sensory deprivation

Because aggrecan protein is modified in a complex manner by post-translational glycosylation (Matthews et al., 2002), detecting aggrecan mRNA by in situ hybridization provides a more direct method to investigate total aggrecan expression. We there- 

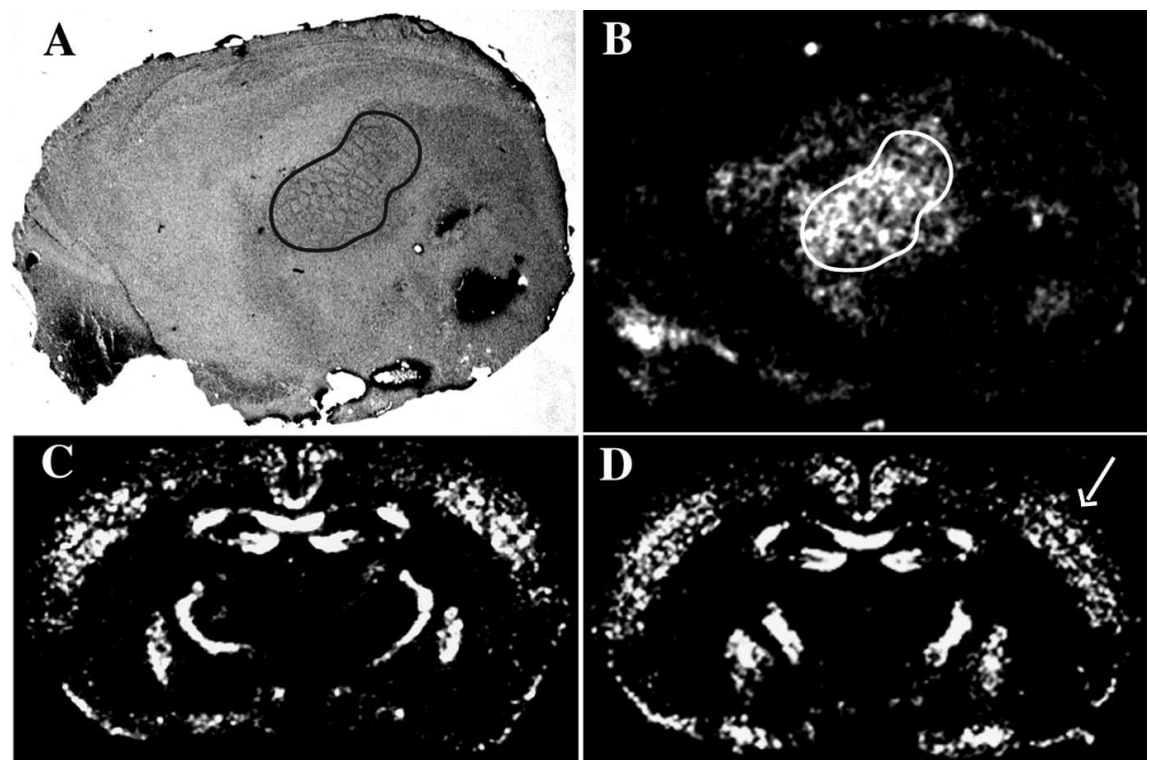

Figure 4. aggrecan $m R N A$ is expressed by neurons in the mouse barrel cortex and altered by sensory deprivation. $\boldsymbol{A}$, Tangential section through layer IV of the barrel cortex stained with cresyl violet to outline the barrel structure. $\boldsymbol{B}$, In situ hybridization, using a ${ }^{33} \mathrm{P}$-labeled antisense probe to exon 12, was performed on the same section, and aggrecan mRNA expression is both enriched and localized within the boundaries of the barrel cortex. $C$, aggrecan mRNA is more enriched in the barrel cortex than neighboring cortical areas. There is a laminar distribution with aggrecan expression more prominent in layers IV and VI of the barrel cortex. $\boldsymbol{D}$, There is a decrease in aggrecan mRNA in the sensory-deprived barrel cortex relative to the nondeprived barrel cortex of the same animal after $30 \mathrm{~d}$ of sensory deprivation during development (the arrow points out the sensory-deprived barrel cortex). The aggrecan expression levels are normal in the nondeprived hemisphere of the trimmed animal. The sensory-deprived barrel cortex shows a decrease in aggrecan message in layer IV, whereas layer VI expression levels appear normal.
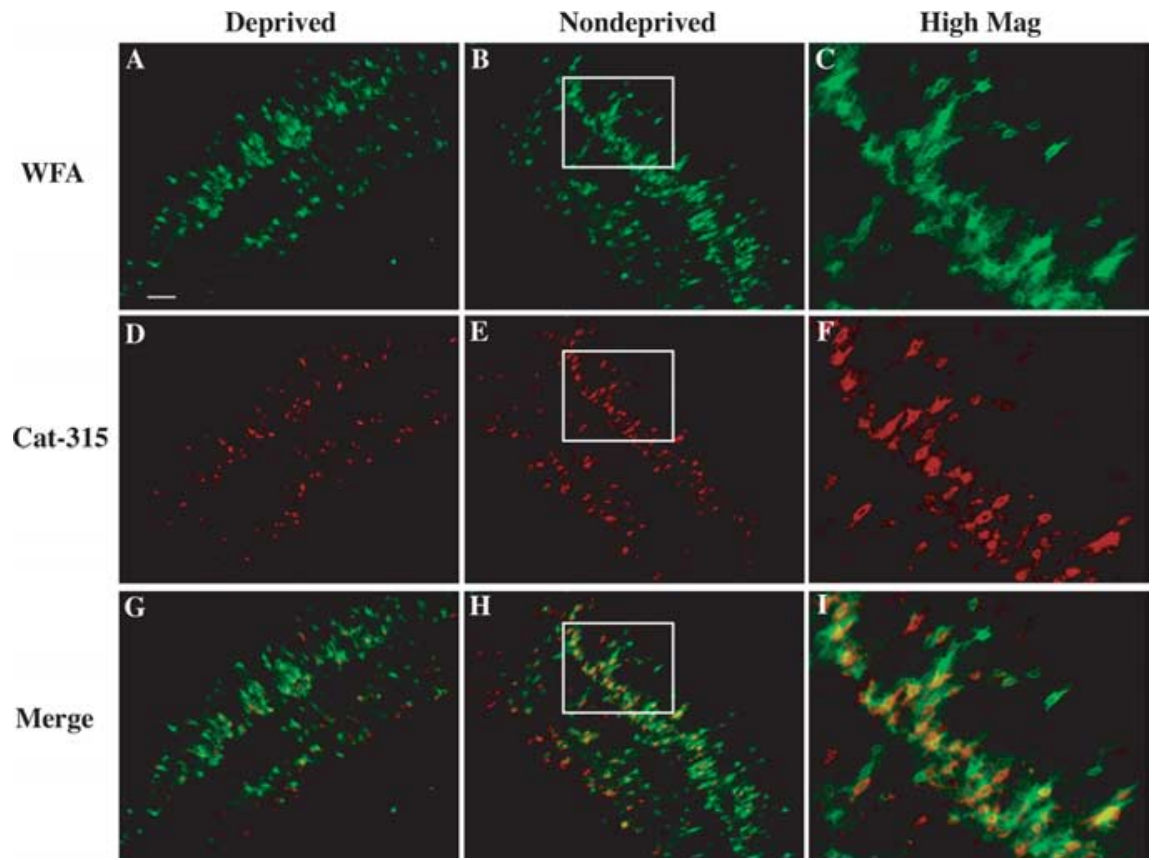

Figure 5. Effects of sensory deprivation on PNs detected with WFA in the mouse barrel cortex. $\mathbf{A}-\mathbf{C}, \mathbf{G}-\mathbf{I}$, In coronal sections, WFA (green) is immunoreactive in layers IV and VI of the mouse somatosensory cortex. $\boldsymbol{A}, \boldsymbol{B}, \boldsymbol{G}, \boldsymbol{H}$, Trimming the whiskers does not alter WFA expression in either the sensory-deprived barrel cortex $(\boldsymbol{A}, \boldsymbol{G})$ or the nondeprived barrel cortex $(\boldsymbol{B}, \boldsymbol{H}) . \boldsymbol{D}, \boldsymbol{E}, \boldsymbol{G}, \boldsymbol{H}$, Trimming leads to reduced expression of Cat-315 (red) in the deprived barrel cortex $(\boldsymbol{D}, \boldsymbol{G})$ but not in the nondeprived barrel cortex $(\boldsymbol{E}, \boldsymbol{H}) . \boldsymbol{C}, \boldsymbol{F}, \boldsymbol{I}$, High-magnification images from the boxed regions show that Cat-315 and WFA have partial levels of overlap but are identifying distinct subsets of cells. Scale bar, $100 \mu \mathrm{m}$.

fore investigated expression of aggrecan mRNA after sensory deprivation to understand whether the changes we observed with Cat-315 were attributable to specific alterations in specific isoforms of the aggrecan protein or are caused by a global loss of aggrecan message. Animals were deprived of sensory input for the first $30 \mathrm{~d}$ of life by having their whiskers unilaterally trimmed. The relative density of autoradiograms was analyzed by densitometry and revealed a significant $17 \%$ decrease $(p=0.0027)$ in aggrecan mRNA expression in layer IV of the deprived barrel cortex compared with the nondeprived barrel cortex (Fig. 4D). Conversely, there was no obvious change in aggrecan message in layer VI of the barrel cortex $(p=0.1385)$. These data are in accord with the decreases we observed in the aggrecan protein, using the Cat-315 antibody, after the same sensory-deprivation paradigm. Together, this suggests that aggrecan expression, at both the protein level and the mRNA level, is activity dependent and requires normal sensory stimulation.

WFA, another PN marker, expression is not altered by sensory deprivation

Our work above describes the specific loss of PNs detected with the antibody Cat-315 and a corresponding reduction in aggrecan mRNA attributable to whisker trimming. However, because Cat-315 detects only of subset of PNs, we now wanted to determine whether PNs detected with other reagents in the rodent barrel cortex were also reduced after whisker trimming. To investigate this, we used WFA, which is a lectin that binds the sugar $\mathrm{N}$-acetlygalactosamine (a component of chondroitin sulfate chains) and has broad reactivity for PNs (Brauer et al., 1993; Bruckner et al., 1993, 1996). Neurons in multiple layers of somatosensory cortex are strongly detected by WFA. Similar to Cat-315, PNs were more abundantly expressed in the barrel cortex region of somatosensory cortex. Of special interest, neurons in layers IV and VI showed the highest density of labeled cells. Surprisingly, there were no significant changes in WFA expression after sensory deprivation (Fig. $5 A-C, G-I$ ) despite the obvious reduction in Cat-315-positive PNs in the deprived barrel cortex compared with the nondeprived barrel cortex (Fig. 5D-F, GI). Stereological counts of WFA-positive cells did not show any tendency toward a decreased number of cells with PNs for the deprived (10.55395 \pm 1.33887$)$ versus the nondeprived $(9.68674 \pm 0.83163 ; n=8$; $p=0.107)$ side. Cat-315 and WFA are in close proximity to one another and occasionally colocalize on the surface of the same neurons, but they generally detect distinct subpopulations of neurons (Fig. 5C, F,I). Therefore, it is critical to define the differences between the subset of cells detected by Cat- 315 versus WFA. 
Cat-315 forms PNs around a subset of parvalbuminexpressing interneurons in layer IV of the barrel cortex To understand why Cat-315 appears to be specifically altered by sensory deprivation, we first needed to molecularly define the subset of cells with Cat-315-reactive PNs in the normal brain. Previous work has suggested that in the cerebral cortex, PNs are localized on a subset of GABAergic interneurons (Celio and Chiquet-Ehrismann, 1993; Wintergerst et al., 1996; Schuppel et al., 2002). Here, we focused on layer IV of the barrel cortex to determine whether aggrecan, detected with Cat-315, defines a known molecular and functional subclass of cells within this region. To investigate this, we double labeled sections with Cat-315 and either anti-parvalbumin or anti-somatostatin antibodies, both of which have been shown previously to detect a functionally distinct subset of interneurons in the cortex (Kawaguchi and Kondo, 2002). We found that all cells that had Cat-315-positive nets in layer IV were parvalbumin positive (Fig. $6 A, C, E, G$ ), although this relationship did not appear to be present in layer VI. Furthermore, the vast majority of parvalbumin-positive cells in layer IV were also Cat-315 positive. In no case were Cat-315positive cells also somatostatin positive in layer IV (data not shown). PNs detected with WFA, which are not altered by sensory deprivation and do not colocalize with Cat-315-expressing PNs, rarely colocalize with parvalbumin-expressing cells in layer IV of the barrel cortex (Fig. 7A-C). Therefore, within the boundaries of layer IV of the barrel cortex, Cat-315 detects a distinct subpopulation of interneurons.

\section{A subset of parvalbumin-expressing cells loses its Cat-315- positive $\mathrm{PN}$ as a result of sensory deprivation}

Sensory deprivation during development leads to a significant decrease in the number of cells with Cat-315-positive PNs, and Cat-315 detects PNs around parvalbumin-expressing interneurons in layer IV of the barrel cortex. Therefore, one explanation for our findings would be that whisker trimming led to a reduction in the number of parvalbumin-positive cells, which would necessarily lead to a reduction in Cat-315-reactive PNs. To determine whether this was a possible explanation for our findings, we again performed unilateral whisker trimming on mice for the first $30 \mathrm{~d}$ of life, after which the barrel cortex was analyzed. Importantly, whisker trimming for the first $30 \mathrm{~d}$ of life did not significantly alter the number of parvalbumin-expressing cells (Fig. $6 A, B)(p=0.8372)$. Despite having no affect on parvalbumin expression, whisker trimming again led to a specific decrease in the number Cat-315-immunoreactive PNs surrounding parvalbumin-positive cells in the deprived barrel cortex compared with the nondeprived barrel cortex (Fig. $6 C, D$ ). These findings are exemplified by the appearance of parvalbumin-positive cells in layer IV of the barrel cortex not ensheathed with a Cat315-positive PN in the sensory-deprived barrel region (Fig. 6E$H)$. Cells with this expression profile are rarely found in the normal barrel cortex. Together, these data indicate that sensory deprivation leads to a specific decrease in aggrecan message and protein in and around parvalbumin-expressing neurons in layer IV of the barrel cortex, resulting in a significant decrease in Cat315-positive PNs. These data are consistent with work done in some laboratories (Maier and McCasland, 1997) but differ from work in other laboratories (Jiao et al., 2006). Differences between studies likely reflect the different paradigms used.

\section{Discussion}

In the present study, we demonstrate that the expression of PNs in the mouse barrel cortex detected by the monoclonal antibody

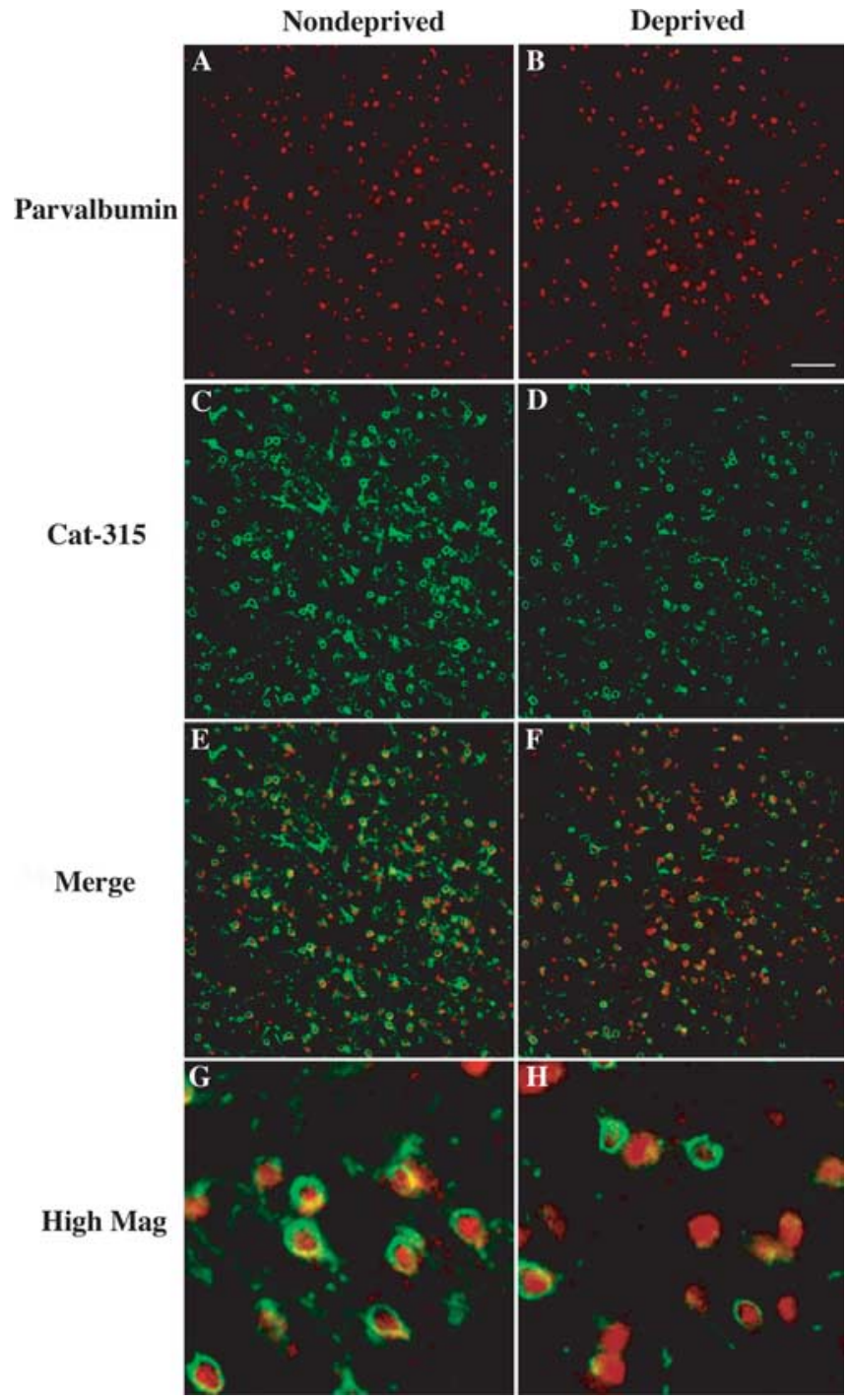

Figure 6. Effects of sensory deprivation on Cat-315-immunoreactive PNs surrounding parvalbumin-expressing interneurons in the mouse barrel cortex. In tangential sections, parvalbumin (red) and Cat-315 (green) were used to stain layer IV of both the nondeprived barrel cortex $(\boldsymbol{A}, \boldsymbol{C}, \boldsymbol{E}, \boldsymbol{G})$ and the sensory-deprived barrel cortex $(\boldsymbol{B}, \boldsymbol{D}, \boldsymbol{F}, \boldsymbol{H})$ of trimmed animals. $\boldsymbol{A}, \boldsymbol{B}$ There is no change in the level of parvalbumin reactivity in the nondeprived barrel cortex $(\boldsymbol{A})$ relative to the deprived barrel cortex $(\boldsymbol{B})$. C, D, Sensory deprivation does not alter Cat-315 expression in the nondeprived barrel cortex $(\boldsymbol{C})$ but does lead to a reduction in Cat-315 expression in the deprived barrel cortex (D). C, E, G, In the nondeprived barrel cortex, Cat-315 forms PNs in the extracellular space around parvalbumin-immunoreactive cells. $\boldsymbol{D}, \boldsymbol{F}, \boldsymbol{H}$, In the sensory-deprived hemisphere, there are parvalbumin-positive interneurons not ensheathed by Cat-315. Scale bar, $100 \mu \mathrm{m}$.

Cat-315, a marker of aggrecan, is dependent on appropriate sensory stimulation during development. Furthermore, we show that aggrecan expression and Cat-315-positive PN expression require sensory stimulation during a critical period within the first $30 \mathrm{~d}$ after birth. Sensory deprivation within this critical period decreases the number of PNs detected with Cat-315 specifically around parvalbumin-expressing cells in layer IV of the barrel cortex. To our knowledge, this is the first demonstration that activity alters $\mathrm{PN}$ expression on a specific subpopulation of cells in the somatosensory cortex.

\section{Cat-315 expression is dependent on normal sensory input} during a critical period in development

PNs first appear in the rodent brain after the second postnatal week, and adult-like expression occurs between P21 and P35 
Parvalbumin
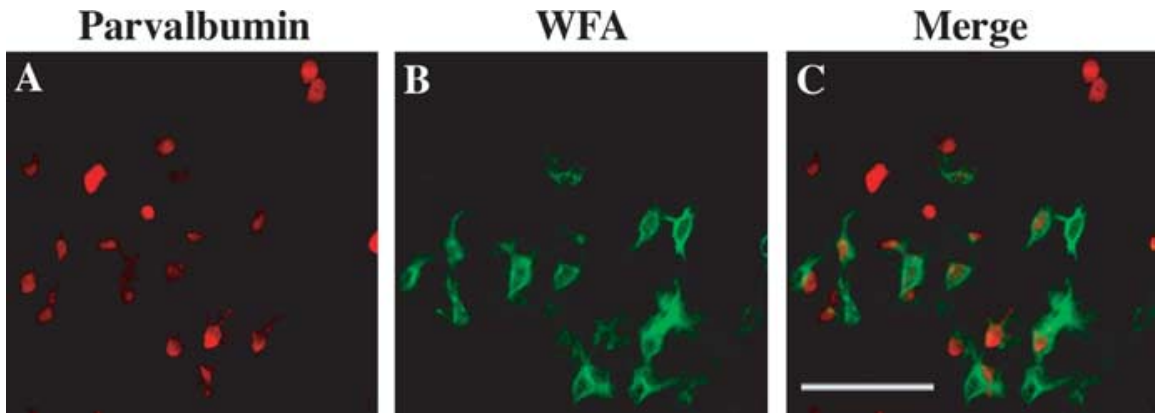

Figure 7. WFA-positive PNs are rarely colocalized with parvalbumin-expressing interneurons in layer IV of the barrel cortex. $\boldsymbol{A}$, Coronal section showing PNs detected with WFA in layer IV of the barrel cortex. $\boldsymbol{B}$, Parvalbumin-immunoreactive cells found in layer IV of the barrel cortex. C, A merged image shows that the majority of WFA-positive cells do not express the calcium-binding protein parvalbumin. Scale bar, $100 \mu \mathrm{m}$.

(Koppe et al., 1997b; Bruckner et al., 2000); their appearance is coincident with aggrecan expression (Matthews et al., 2002). In layer IV of the barrel cortex, PNs first appear around P10, coincident with the decreased plasticity that takes place after the first postnatal week (Stern et al., 2001; Fox, 2002) and the onset of whisking.

The somatosensory cortex requires appropriate peripheral activity during an early critical period for proper cortical patterning (Huntley, 1997). Here, we demonstrate that the number of cells bearing PNs, detected with the antibody Cat-315, in layer IV of the mouse barrel cortex is significantly decreased as a result of sensory deprivation through $\mathrm{P} 30$. These data suggest that the development and expression of Cat-315-positive PNs is activity dependent and requires normal activation of the barrel cortex.

Sensory deprivation in adults did not alter Cat-315 expression in the barrel cortex, indicating that once established, maintenance of PNs does not require normal sensory input. This is consistent with observations in the cat visual system, where Cat301 expression, also a marker of aggrecan, decreases after sensory deprivation during development but not in adulthood (Sur et al., 1988). Because sensory deprivation during the first $30 \mathrm{~d}$ of life but not in adulthood led to decreased Cat-315 expression, there could be a critical period during development when normal sensory input is required for $\mathrm{PN}$ formation.

To better understand the critical period for PN development, we studied animals that experienced $30 \mathrm{~d}$ of sensory deprivation, followed by $30 \mathrm{~d}$ of normal stimulation. In this group, we found a significant decrease in Cat-315 expression demonstrating a persistent and irreversible effect of abnormal sensory experience. These data indicate that trimming for $30 \mathrm{~d}$ did not simply delay Cat-315-positive PN development and also suggest that the close of the critical period for Cat-315-reactive PN development is at or before P30 in the barrel cortex.

Interestingly, the decrease in Cat-315 was more apparent in the animals in which whiskers were allowed to regrow than in animals killed at P30. Although somewhat surprising, these data may simply reflect the continuing maturation of PNs, which structurally evolve into adulthood (Koppe et al., 1997b).

Cat-315 detects a carbohydrate epitope on a glycoform of aggrecan in a subset of PNs (Matthews et al., 2002; Dino et al., 2006). Therefore, we investigated whether whisker trimming led to a specific loss of aggrecan mRNA or a decrease in the specific glycoform of aggrecan detected by Cat-315. Interestingly, there was a $17 \%$ decrease in aggrecan mRNA in layer IV of the deprived barrel cortex compared with the nondeprived barrel cortex. This decrease is in accord with the $22 \%$ decrease we detected in aggre- can protein expression. Together, these data indicate that expression of the aggrecan protein and message in the mouse barrel cortex are activity dependent.

\section{Sensory deprivation leads to the loss of a} particular subset of PNs in the barrel cortex

As described above, our work showed that PNs, detected with Cat-315, were reduced by whisker trimming and that this reduction is likely mediated by a reduction in the expression of aggrecan mRNA. Because previous work has shown that Cat-315 only detects a subset of aggrecan protein, we expected that we would detect a reduction in PNs with other markers. We investigated this hypothesis with the lectin WFA. WFA is widely used as a broad PN marker (Hartig et al., 1994; Koppe et al., 1997a,b; Bruckner et al., 1998; Preuss et al., 1998) because it binds to CSPGs (Bruckner et al., 1996; Koppe et al., 1997a). Surprisingly, PNs detected with WFA in the barrel cortex were not altered by whisker trimming. Additional analysis showed that in normal somatosensory cortex, Cat-315 and WFA detect a somewhat overlapping but clearly nonidentical subset of cells in the mouse barrel cortex. These data indicate that in the barrel cortex, WFA detects only a subset of PNs and CSPGs. Furthermore, these data suggest that a subpopulation of cells with PNs, specifically detected by Cat-315, is uniquely affected by sensory deprivation.

An alternative interpretation is that PNs are still present but are no longer detected by Cat-315 because of a loss of aggrecan and/or change in aggrecan glycosylation. We attempted to investigate this by using reagents that have broad reactivity for CSPGs (including lectins and "stub" antibodies). However, we found no reagent that demonstrated that $\mathrm{PNs}$ remain after deprivation (data not shown). This has lead to the conclusion that the molecular composition of PNs has not simply changed but they are, in fact, lost with deprivation. It is, however, possible that PNs still remain after deprivation but that their molecular nature has changed, making them undetectable by any of the reagents that we used in this study.

Here, we have demonstrated that in layer IV of the mouse barrel cortex, Cat-315 is a specific marker of parvalbuminpositive interneurons. In this study, we clearly demonstrate that without proper sensory input, aggrecan forms significantly fewer PNs, specifically around parvalbumin-expressing cells in layer IV of the barrel cortex, whereas parvalbumin expression itself is unaffected. It is this subset of parvalbumin cells that requires normal sensory activity to express Cat-315-positive PNs.

\section{Functional implications of PN loss on the developing barrel cortex}

The function of the PN is not fully understood. Previous work, however, has suggested that the formation of PNs signals the close of the critical period and has a role in synapse stabilization (Guimaraes et al., 1990; Hockfield et al., 1990). Pizzorusso et al. (2002) demonstrated that PN expression correlates with the close of the critical period of the visual system, and their work strongly suggests a role for the PNs in reducing plasticity, indicative of a shift from an immature plastic state cortex to a mature cortex. The PN also appears at the close of the critical period for layer IV of the barrel cortex (Koppe et al., 1997b), where most of the 
developmental processes end during the first postnatal month (Fox, 1992; Glazewski and Fox, 1996).

Previous work has established the importance of the development of local inhibitory circuits in developmental plasticity and regulating the closing of the critical period (for review, see Hensch, 2005). Of particular interest are the parvalbumin-expressing interneurons, the emergence of which correlates most closely with decreased synaptic plasticity and the close of the critical period (Del Rio et al., 1994). Interestingly, previous work has demonstrated that parvalbumin-positive cells are frequently surrounded by PNs (Kosaka and Heizmann, 1989), which has lead investigators to suggest that this structure may contribute to the ability of these cells to maintain high levels of neuronal discharge (Hartig et al., 1999; Morris and Henderson, 2000). Interestingly, the population of parvalbumin-expressing cells in the cortex is defined by at least two distinct cellular subtypes, basket cells and chandelier cells (DeFelipe et al., 1989). Because we found altered Cat-315-positive PN expression after sensory deprivation in a subset of parvalbumin-positive cells $(22 \%$ at $30 \mathrm{~d}$ and $48 \%$ at $60 \mathrm{~d}$ ), it raises the question whether any one of these cellular subtypes is uniquely affected by deprivation. However, in the layer IV of the barrel cortex, it is likely that the majority of parvalbumin-positive cells are basket cells because chandelier cells, although abundant in layer II/III, are expressed sparingly in deeper layers of primary sensory cortices including somatosensory cortex and visual cortex of rat, monkey, and human (Peters et al., 1982; DeFelipe et al., 1989, 1998; Inda et al., 2007). Because we ultimately find alteration of nets on nearly $50 \%$ of the parvalbumin-positive cells in layer IV, this argues strongly for alteration of Cat-315-positive nets predominantly on basket cells. Consistent with this, previous work has shown that although basket cells are coated with PNs, chandelier cells are not (Naegle and Katz, 1990).

Identification of the subcellular subtype has important functional implications. Basket cells extend a horizontal plexus making contacts on the somas of target cells; in contrast, chandelier cells make powerful "cartridge" synapses onto the axons of target cells. Much recent work has suggested a particularly important role for basket cells in the close of the critical period, whereas a more important role for chandelier cells is controlling excessive excitation and spike timing in the cortex. Therefore, we would predict that altered net expression on basket cells in deprived animals would lead to decreased perisomatic inhibition within cortical barrels and delayed closure of the critical period. Interestingly, these predictions are consistent with previous work showing that whisker trimming during development leads to increased excitability and decreased inhibition in layer IV of the somatosensory system (Simons and Land, 1987; Shoykhet et al., 2005). In addition, these data are in agreement with previous work demonstrating that disruption of molecular components of PNs leads to a reduction in perisomatic inhibition (Saghatelyan et al., 2001), reopening of the critical period (Pizzorusso et al., 2002, 2006), and that dark rearing, which delays the close of the critical period, inhibits expression of aggrecan-positive PNs identified with Cat-301 (Guimaraes et al., 1990; Hockfield et al., 1990). We cannot, however, exclude the possibility that alteration of nets on chandelier cells also occurred in this study. We presently do not know what defines the specific subset of parvalbumin cells on which nets were altered. Future work will attempt to address these questions.

We demonstrate that the expression of the aggrecan within PNs is dependent on activity in the barrel cortex because both the protein markers and mRNA are decreased after sensory depriva- tion during the critical period. These results are comparable to studies in the cat visual system that showed a reduction in PN markers with sensory deprivation (Hockfield and McKay, 1983; Sur et al., 1988; Guimaraes et al., 1990; Lander et al., 1997), which were later shown to be specific markers of aggrecan expression (Matthews et al., 2002). However, this study represents the first direct demonstration that aggrecan is expressed in an activitydependent manner and directly contributes to the development of the PN.

\section{References}

Brauer K, Hartig W, Bigl V, Bruckner G (1993) Distribution of parvalbumin-containing neurons and lectin-binding perineuronal nets in the rat basal forebrain. Brain Res 631:167-170.

Bruckner G, Brauer K, Hartig W, Wolff JR, Rickmann MJ, Derouiche A, Delpech B, Girard N, Oertel WH, Reichenbach A (1993) Perineuronal nets provide a polyanionic, glia-associated form of microenvironment around certain neurons in many parts of the rat brain. Glia 8:183-200.

Bruckner G, Bringmann A, Koppe G, Hartig W, Brauer K (1996) In vivo and in vitro labelling of perineuronal nets in rat brain. Brain Res 720:84-92.

Bruckner G, Bringmann A, Hartig W, Koppe G, Delpech B, Brauer K (1998) Acute and long-lasting changes in extracellular-matrix chondroitinsulphate proteoglycans induced by injection of chondroitinase $\mathrm{ABC}$ in the adult rat brain. Exp Brain Res 121:300-310.

Bruckner G, Grosche J, Schmidt S, Hartig W, Margolis RU, Delpech B, Seidenbecher CI, Czaniera R, Schachner M (2000) Postnatal development of perineuronal nets in wild-type mice and in a mutant deficient in tenascin-R. J Comp Neurol 428:616-629.

Celio MR, Blumcke I (1994) Perineuronal nets-a specialized form of extracellular matrix in the adult nervous system. Brain Res Brain Res Rev 19:128-145.

Celio MR, Chiquet-Ehrismann R (1993) "Perineuronal nets" around cortical interneurons expressing parvalbumin are rich in tenascin. Neurosci Lett 162:137-140.

DeFelipe J, Gonzalez-Albo MC (1998) Chandelier cell axons are immunoreactive for GAT-1 in the human neocortex. NeuroReport 9:467-470.

DeFelipe J, Hendry SH, Jones EG (1989) Visualization of chandelier cell axons by parvalbumin immunoreactivity in monkey cerebral cortex. Proc Natl Acad Sci USA 86:2093-2097.

Del Rio JA, De Lecea L, Ferrer I, Soriano E (1994) The development of parvalbumin-immunoreactivity in the neocortex of the mouse. Dev Brain Res 81:247-259.

Dino MR, Harroch S, Hockfield S, Matthews RT (2006) Monoclonal antibody Cat-315 detects a glycoform of receptor protein tyrosine phosphatase beta/phosphacan early in CNS development that localizes to extrasynaptic sites prior to synapse formation. Neuroscience 142:1055-1069.

Fox K (1992) A critical period for experience-dependent synaptic plasticity in rat barrel cortex. J Neurosci 12:1826-1838.

Fox K (2002) Anatomical pathways and molecular mechanisms for plasticity in the barrel cortex. Neuroscience 111:799-814.

Glazewski S, Fox K (1996) Time course of experience-dependent synaptic potentiation and depression in barrel cortex of adolescent rats. J Neurophysiol 75:1714-1729.

Guimaraes A, Zaremba S, Hockfield S (1990) Molecular and morphological changes in the cat lateral geniculate nucleus and visual cortex induced by visual deprivation are revealed by monoclonal antibodies Cat-304 and Cat-301. J Neurosci 10:3014-3024.

Hartig W, Brauer K, Bigl V, Bruckner G (1994) Chondroitin sulfate proteoglycan-immunoreactivity of lectin-labeled perineuronal nets around parvalbumin-containing neurons. Brain Res 635:307-311.

Hartig W, Derouiche A, Welt K, Brauer K, Grosche J, Mader M, Reichenbach A, Bruckner G (1999) Cortical neurons immunoreactive for the potassium channel Kv3.1b subunit are predominantly surrounded by perineuronal nets presumed as a buffering system for cations. Brain Res 842:15-29.

Hensch TK (2005) Critical period plasticity in local cortical circuits. Nat Rev Neurosci 6:877-888.

Hockfield S, McKay R (1983) Monoclonal antibodies demonstrate the organization of axons in the leech. J Neurosci 3:369-375.

Hockfield S, Kalb RG, Zaremba S, Fryer H (1990) Expression of neural pro- 
teoglycans correlates with the acquisition of mature neuronal properties in the mammalian brain. Cold Spring Harb Symp Quant Biol 55:505-514.

Huntley GW (1997) Differential effects of abnormal tactile experience on shaping representation patterns in developing and adult motor cortex. J Neurosci 17:9220-9232.

Inda MC, Defelipe J, Munoz A (2007) The distribution of chandelier cell axon terminals that express the GABA plasma membrane transporter GAT-1 in the human neocortex. Cereb Cortex, in press.

Jiao Y, Zhang C, Yanagawa Y, Sun QQ (2006) Major effects of sensory experiences on the neocortical inhibitory circuits. J Neurosci 26:8691-8701.

Kawaguchi Y, Kondo S (2002) Parvalbumin, somatostatin and cholecystokinin as chemical markers for specific GABAergic interneuron types in the rat frontal cortex. J Neurocytol 31:277-287.

Kind PC, Beaver CJ, Mitchell DE (1995) Effects of early periods of monocular deprivation and reverse lid suture on the development of Cat-301 immunoreactivity in the dorsal lateral geniculate nucleus (dLGN) of the cat. J Comp Neurol 359:523-536.

Koppe G, Bruckner G, Hartig W, Delpech B, Bigl V (1997a) Characterization of proteoglycan-containing perineuronal nets by enzymatic treatments of rat brain sections. Histochem J 29:11-20.

Koppe G, Bruckner G, Brauer K, Hartig W, Bigl V (1997b) Developmental patterns of proteoglycan-containing extracellular matrix in perineuronal nets and neuropil of the postnatal rat brain. Cell Tissue Res 288:33-41.

Kosaka T, Heizmann CW (1989) Selective staining of a population of parvalbumin-containing GABAergic neurons in the rat cerebral cortex by lectins with specific affinity for terminal $\mathrm{N}$-acetylgalactosamine. Brain Res 483:158-163.

Lander C, Kind P, Maleski M, Hockfield S (1997) A family of activitydependent neuronal cell-surface chondroitin sulfate proteoglycans in cat visual cortex. J Neurosci 17:1928-1939.

Maier DL, McCasland JS (1997) Calcium-binding protein phenotype defines metabolically distinct groups of neurons in barrel cortex of behaving hamsters. Exp Neurol 145:71-80.

Matthews RT, Kelly GM, Zerillo CA, Gray G, Tiemeyer M, Hockfield S (2002) Aggrecan glycoforms contribute to the molecular heterogeneity of perineuronal nets. J Neurosci 22:7536-7547.

Morris NP, Henderson Z (2000) Perineuronal nets ensheath fast spiking, parvalbumin-immunoreactive neurons in the medial septum/diagonal band complex. Eur J Neurosci 12:828-838.

Naegele JR, Katz LC (1990) Cell surface molecules containing $\mathrm{N}$-acetylgalactosamine are associated with basket cells and neurogliaform cells in cat visual cortex. J Neurosci 10:540-557.

Peters A, Proskauer CC, Ribak CE (1982) Chandelier cells in rat visual cortex. J Comp Neurol 206:397-416.
Pizzorusso T, Medini P, Berardi N, Chierzi S, Fawcett JW, Maffei L (2002) Reactivation of ocular dominance plasticity in the adult visual cortex. Science 298:1248-1251.

Pizzorusso T, Medini P, Landi S, Baldini S, Berardi N, Maffei L (2006) Structural and functional recovery from early monocular deprivation in adult rats. Proc Natl Acad Sci USA 103:8517-8522.

Preuss TM, Gray D, Cusick CG (1998) Subdivisions of the motor and somatosensory thalamus of primates revealed with Wisteria floribunda agglutinin histochemistry. Somatosens Mot Res 15:211-219.

Saghatelyan AK, Dityatev A, Schmidt S, Schuster T, Bartsch U, Schachner M (2001) Reduced perisomatic inhibition, increased excitatory transmission, and impaired long-term potentiation in mice deficient for the extracellular matrix glycoprotein tenascin-R. Mol Cell Neurosci 17:226-240.

Schuppel K, Brauer K, Hartig W, Grosche J, Earley B, Leonard BE, Bruckner G (2002) Perineuronal nets of extracellular matrix around hippocampal interneurons resist destruction by activated microglia in trimethyltintreated rats. Brain Res 958:448-453.

Shoykhet M, Land PW, Simons DJ (2005) Whisker trimming begun at birth or on postnatal day 12 affects excitatory and inhibitory receptive fields of layer IV barrel neurons. J Neurophysiol 94:3987-3995.

Simons DJ, Land PW (1987) Early experience of tactile stimulation influences organization of somatic sensory cortex. Nature 326:694-697.

Stern EA, Maravall M, Svoboda K (2001) Rapid development and plasticity of layer 2/3 maps in rat barrel cortex in vivo. Neuron 31:305-315.

Sur M, Frost DO, Hockfield S (1988) Expression of a surface-associated antigen on Y-cells in the cat lateral geniculate nucleus is regulated by visual experience. J Neurosci 8:874-882.

West MJ, Slomianka L, Gundersen HJ (1991) Unbiased stereological estimation of the total number of neurons in the subdivisions of the rat hippocampus using the optical fractionator. Anat Rec 231:482-497.

Wiesel TN, Hubel DH (1963a) Effects of visual deprivation on morphology and physiology of cells in the cats lateral geniculate body. J Neurophysiol 26:978-993.

Wiesel TN, Hubel DH (1963b) Single-cell responses in striate cortex of kittens deprived of vision in one eye. J Neurophysiol 26:1003-1017.

Wintergerst ES, Vogt Weisenhorn DM, Rathjen FG, Riederer BM, Lambert S, Celio MR (1996) Temporal and spatial appearance of the membrane cytoskeleton and perineuronal nets in the rat neocortex. Neurosci Lett 209:173-176.

Yamaguchi Y (2000) Lecticans: organizers of the brain extracellular matrix. Cell Mol Life Sci 57:276-289.

Zaremba S, Guimaraes A, Kalb RG, Hockfield S (1989) Characterization of an activity-dependent, neuronal surface proteoglycan identified with monoclonal antibody Cat-301. Neuron 2:1207-1219. 